\title{
Research on Control Characteristics of Three Axis Paraleel Mechanism Based on Pic16f84a
}

\author{
1, ${ }^{*}$ Ing. Alvaro Ruddy Miranda Torrez, ${ }^{2}$ Gan Xinji \\ ${ }^{1}$ Mechanical Engineering Institute, Beihua University, Jilin City, Jilin Province, 132021, China \\ ${ }^{2}$ School of Mechanical Engineering, Beihua University, Jilin, 132021, China \\ *alvaromt_14@hotmail.com
}

Keywords: Engineering, microcontroller, parallel manipulator, PIC, programing, Proteus.

\begin{abstract}
This paper is about a parallel manipulator and how to control it using a microcontroller and c++ language. It's explained which devices are necessary and why are the optimum for this project. Everything is followed by a simulation using a computer software.
\end{abstract}

\section{Introduction}

Parallel manipulators have attracted the attention in research institutions and industry due to their high theoretical dynamics potential, structural rigidity and high accuracy due to the closed kinematic loops and no error accumulating characteristics. Also could be named as hexapod, a Stewart platform, Gough platform, Stewart-Gough platform, a parallel kinematic machine (PKM) or a parallel robot.The first prototypes of parallel kinematics machine (PKM) tools were introduced to the public in 1994 by Ingersoll and Ginddings \& Lewis. During the last decade, according more than 100 different parallel mechanical architectures have been built, mostly as prototypes or academic studies. Although more than 15 years passed since the first commercial parallel kinematics machine tools were introduced, they are not widely accepted in the industry. There are two reasons for that. First, from the beginning of their appearance it became obvious that implementation of their theoretical capabilities in practice introduces many new problems. The second reason is the psychological arguments like:

- lack of trust in the new, strange looking technology,

- reluctance to be first to try out the new technology,

- lack of accepted standards for assessing the users value of the parallel kinematics machine tools.

\section{Characteristics of the Three Axis Parallel Mechanism Nowdays}

Compared with conventional machine tools, parallel kinematic machine (PKM) has many advantages, for example, higher stiffness and higher force-to-weight ratio. This is regarded as a revolutionary concept for machine tools.

Many research works about diverse aspects of PKM have been published but they are still $\mathrm{R} \& \mathrm{D}$ topic in many laboratories. 


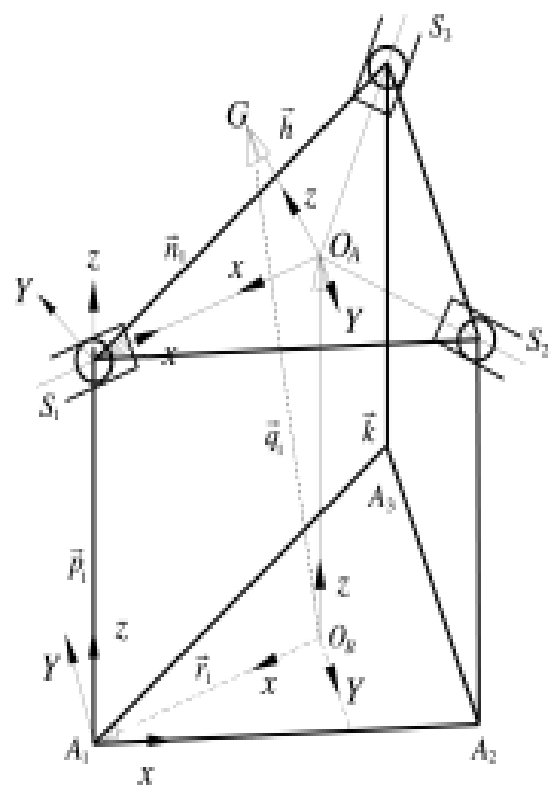

Figure 1. 3-PSP parallel manipulator based on chute-type spherical joint.

It is well-known that the shape and volume of the workspace are one of the greatest weaknesses of parallel kinematic machine tools. Hexaglide and Triaglide mechanisms are examples where workspace extension is achieved by elongating one axis as a principal motion axis that is a common feature of all Cartesian machines. With the idea of principal axis of motion in mind, a new 3-dof spatial parallel mechanism for horizontal and vertical milling machines has been developed. The mechanism consists of the mobile platform, three joint parallelograms c1, c2 and c3, and a stationary base with two parallel guideways.

Two crossed parallelograms $\mathrm{c} 1$ and $\mathrm{c} 2$, with spherical and/or universal, i.e., cardan joints, are connected with one of their ends to the mobile platform, and with their other ends to the independent sliders s1 and s2 which, with a common guideway, make two powered and controlled translatory joints.

The third joint parallelogram $\mathrm{c} 3$ is connected with one of its ends, through passive rotating and translatory joints to the mobile platform. Its other end is connected with rotating joints to the slider s3, which makes, with the second guideway, the third powered and controlled translatory joint. The actuation of sliders s1, s2 and s3 offers three degrees of freedom to the mobile platform, i.e., the tool, so that the platform in its motion through the space retains constant orientation.

The influence of mechanism structure on the workspace shape and volume is reflected in the following

The parallel guideways provide:

- arbitrary workspace length in $\mathrm{X}$ direction, and regular workspace shape on its boundaries in $\mathrm{Y}$ direction, i.e., Ymin $=$ constant and $\mathrm{Ymax}=$ constant.

The crossing of joint parallelograms $\mathrm{c} 1$ and $\mathrm{c} 2$ provides:

- decreasing of guideway lengths for the same X dimension of workspace, and smaller curvature on Xmin and Xmax workspace borders.

Passive translatory dof or joint in $Y$ direction provides:

- decoupling of platform motions in $\mathrm{Z}$ and $\mathrm{Y}$ directions, and - Exceptional workspace regularity with $\mathrm{Zmin}=$ constant and $\mathrm{Zmax}=$ constant on its borders for each of $\mathrm{Ymin}$ $\leq \mathrm{Y} \leq \mathrm{Ymax}$.

In comparison with similar developed mechanisms it has several advantages such as: rather regular shape of the workspace (slightly modified block) similar to serial machines; greater stiffness by nature of struts arrangement; good force and speed ratio through the entire mechanism 
workspace. The variance of mechanism structure and design solutions enables a wide range of applications for vertical and horizontal 3-axis milling machines, as described in, where first developed vertical milling machine prototype has been presented.

\section{A. Classification of parallel kinematics machines}

A parallel manipulator is a closed-loop mechanism in which the end-effector (mobile platform) is connected to the base (fixed platform) by at least two independent kinematic chains. Between the base and end-effector platforms are serial chains (called limbs or legs).

A fully-parallel manipulator is a closed-loop mechanism with an n-DOF end-effector connected to the base by $n$ independent kinematic chains, which have at most two links and they are actuated by a unique prismatic or rotary actuators.

Combinations of fully-parallel manipulator and additional serial axis are referred as hybrid systems.

The main idea is subdivision the mechanism into simple functional units. The kinematic substructures for the generation of the platform joint movements are chosen from a list of predefined solutions. A valid combination of these basic elements as well as the number of drives is then enumerated with respect to the required DOF of the end-effector. The geometric configuration of the joint connections is finally chosen from a list of predefined solutions.

Parallel manipulators can be classified according to their nature of motion in planar, spherical and spatial. The other classification is according the DOF of the end-effector.

The same end-effector movement can be realised with constant length legs but actuated foot points, for example Hexaglide and Linapod as 6-DOF 6-P(RR)S actuated with linear drives, or Hexa robot as 6-DOF 6-R(RR)S actuated with rotary drives. By grouping the individual chains into pairs with a common drive, three degrees of freedom can be locked which results in parallel mechanisms with 3 DOF of the end-effector, examples are Delta robot and Triaglide.

Table 1. Advantages and Disadvantages of parallel machines

\begin{tabular}{||c|c|}
\hline Advantages & Disadvantages \\
\hline High stiffness due to closed-loop kinematic chains & Small and complex workspace \\
\hline Only compression and tension in the struts, no bending forces & Low workspace/machine size ratio \\
\hline Small inertia & Very complex control \\
\hline Very high dynamics performances due to the low moving mass & Very susceptible to thermal loads \\
\hline High payload/ machine weight ratio & Inherent danger of strut collision \\
\hline Many equal components & Singularities in the workspace \\
\hline Possibility for modular design and reconfigurability & Performance is pose dependent \\
\hline Position error-averages & Complex key-components \\
\hline Linear drives used for rotary movements & Complicated calibration \\
\hline Maximum force-summation of all actuator forces & Force error-accumulates \\
\hline
\end{tabular}

B. Parallel machines-advantages and disadvantages

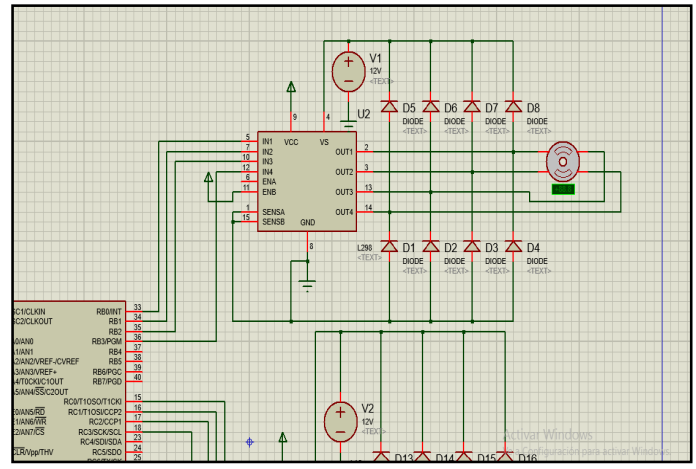

Figure 2. Circuit to control the motors 


\section{Design of the Three Axis Parallel Mechanism Control System Based on PIC16F84A}

The control system for the three-axis paralleling mechanism was previously designed with the help of Proteus software. Where simulations were performed to determine their effectiveness.

Basically the first objective was to control the three bipolar motors, so that they can move in both directions and stop when the operator decides.

The components required for the design of this system were the following:

- Three Haydon 15000 bipolar motors

- Diodes

- L298 which is a dual h-bridge motor driver

- An external source and that the motors operate with $12 \mathrm{~V}$

In addition the controller component of the circuit will be:

- PIC 16F877A

That will need certain components that will help us with starting and stopping the rotation of the engines besides deciding the direction:

- Diodes

- Switches

- Resistors of $10 \mathrm{k}$

And for the correct operation of the PIC is used:

- 22pF Capacitors

- A Crystal- Resistencias de 10k

- Switch

\section{A. Reasons to choose the PIC $16 f 877$ a}

The 16F877 microcontroller CPU as well as the entire PIC family of Microchip are of the RISC (Reduced Instruction Set Computer) type that makes them faster than those known as CISC. The advantage lies in the speed of processing because while reading the program memory can be executing instructions, so when it finishes executing one instruction already has ready the other one without wasting time, in that next cycle of execution. In addition, the program memory instructions are 14-bit words allowing better control and performance of the microcontroller.

All instructions are executed in a single clock cycle because as already mentioned when executing one instruction already has the other ready to be executed without losing a clock cycle. Only the jump instructions take two cycles, one to see where to jump and another to do. Compared to other $\mathrm{uC}$ like the 8051 is very effective, since it has to take into account that the latter has instructions of several clock cycles, are slower because they share the data bus and memory read.

Stack of 8 levels; Each time a CALL subroutine is called, in the stack the previous vector info is saved, thus having the RETURN instructions, the value of that vector is read and it returns to the called point. So you can have eight calls to subroutine and return to the original point without problems.

Up to $20 \mathrm{MHz}$ of speed, this is the maximum speed of the PIC according to the manufacturer, very sufficient for control instructions and for control algorithms that do not reach the DSP, for the latter look for the 18 series Microchip. Some people make the microcontroller run faster than specified using what is called overclocking, from which you can find a lot of information on the network, you should only take into account that its good operation is not guaranteed and may cause failure, everything depends How important your process is and if you really need more speed. 
In addition to the large number of input and output pins it has been a great advantage for this type of applications, otherwise it would have been necessary to use a microcontroller for each motor, but that would not be an effective system.

The L298 drivers are vital for this project, since being the bipolar motors, you need a $\mathrm{H}$ bridge to get these functions properly. In addition to being relatively large engines, we opted to use a power source exclusive to the engines, which is $12 \mathrm{~V}$.

The number of motors is three because each motor belongs to each of the three axes of the mechanism of three parallel axes.

After the design of the circuit has the program for the PIC, this was designed in the programming language $\mathrm{c}++$.

After carrying out the program, simulations were also carried out with the help of the Proteus program, where the correct functioning of the circuit and the program was verified.

Once the simulation is completed, it is possible to go to the physical part of the work, to assemble the circuit and the mechanism, finally to integrate these two parts and to perform the tests of the correct assembly and no longer of the operation since in the step of the simulation were demonstrated that everything is correct.

\section{Circuit and Program's Experiment}

For this project only experiments were carried out in a virtual way, that is to say, through the computer with the help of Proteus software, which is a complete software for this type of project.

In this software is where the design of the circuit step by step, where the appropriate components were chosen and could be optimized to the circuit in every aspect. In addition we have the advantage that Proteus allows us to download the programs compiled to the microcontroller and this way you can see the existence of some error, either in the electronic or control and correct them.

In this way physical experimentation would be avoided where if some mistake is made, the solution would become more expensive in time and resources.

Therefore, after performing a series of simulations, it was observed that both the program and the circuit worked as expected. The three bipolar motors are able to move in both directions and stop with pressing a switch (one for each direction). In addition, all three can work at the same time, even in different directions.

The only drawback is that the chosen PIC cannot control the three motors in parallel, so that the motors cannot be stopped at the same time. However, a stop button was installed, with which for any emergency it is possible to stop the three motors in parallel.

The tests were done and the button works according to the established in the program. 




Figure 3. The complete Control Circuit

After the simulations is the physical circuit and the mechanism, which was not completed due to lack of components.

\section{Conclusions}

After the simulation it can be said that both the program and the design of the circuit are optimal since they proved to work correctly.

It was only a problem that was the handling of the motors in parallel product of the microcontroller that was chosen to carry out the project, however it was solved with the stop button. The operator of the machine is one, which makes it difficult to use more than one engine at a time, you have to start them individually and also stop them. However in case of an emergency and something has gone out of control, this is the stop button that will stop any situation. However, both the program and the three-axis parallel mechanism allow the three motors to operate at the same time making different movements of each other, which is an advantage in this project since if it were a serial mechanism, this task could not be done.

By not performing physical tests, it could be said that the project is not profitable, but even in huge projects, only pilot tests are carried out before the execution of the project. So it can be said that in case there is a problem, this will be produced by the mechanical part of the project that is not included in this work, i.e. the structure of the mechanism, since it is proven that both the program and the design of the Circuit are suitable.

Then we could conclude that with this project we managed to design a circuit which is controlled by a program that manages to operate a system of three parallel axes.

\section{Conflict of Interest}

The author declares that there is no conflict of interest. 


\section{References}

[1] J. Angeles, D. Chablat, On isotropic sets of points in the plane. Application to the design of robot architectures, in: Proc. 7th International Symposium on Advances in Robot Kinematics, Slovenia, 2000.

[2] D. Chablat, P.Wenger, J. Angeles, Conception isotropique d'une morphologie parallèle : application à l'usinage, in: Proc. 3rd International Conference On Integrated Design and Manufacturing in Mechanical Engineering, Montreal, Canada, 2000.

[3] D. Chablat, P. Wenger, A new three-DOF parallel mechanism: milling machine applications, 2nd Chemnitz Parallel Kinematics Seminar, Chemnitz, Germany, 2000.

[4] F. Majou, P. Wenger, D. Chablat, The Design of Parallel Kinematic Machine Tools Using Kinetostatic Performance Criteria, in: Proc. 3rd International Conference on Metal Cutting, Metz, France, 2001.

[5] P. Wenger, D. Chablat, Kinematic Analysis of a New Parallel Machine Tool: The Orthoglide, in: Proc. 7th International Symposium on Advances in Robot Kinematics, Portoroz, Slovenia, 2000.

[6] P. Wenger, D. Chablat, C. Gosselin, A Comparative Study of Parallel Kinematic Architectures for Machining Applications, in: Proc. 2nd Workshop on Computational Kinematics, Seoul, Korea, 2001.

[7] Angeles J., The Qualitative Synthesis of Parallel Manipulators, Proceedings of the WORKSHOP on Fundamental Issues and Future Research Directions for Parallel Mechanisms and Manipulators October 3-4, 2002, Quebec City, Quebec, Canada, 2002, pp. 160-168

[8] E. Annacondia et al., An Experience in Design and Development of Joints for Parallel Kinematics Machines, The 3rd Chemnitz Parallel Kinematics Seminar PKS 2002, Chemnitz, Germany, 2002, pp. 243-261

[9] I. Bonev, Delta Parallel Robot - the Story of Success, 2001. Available: www.parallemic.org/Reviews/Review002.html

[10] I. Bonev, What is Going on With Parallel Robots, 2003. Available: www.roboticsonline.com/public/articles/

[11] T. Brogardh, PKM Research - Important Issues, as seen from a Product Development Perspective at ABB Robotics, Proceedings of the WORKSHOP on Fundamental Issues and Future Research Directions for Parallel Mechanisms and Manipulators October 3-4, 2002, Quebec City, Quebec, Canada, 2002, pp. 68-82.

[12] M. Ceccarelli, G. Carbone, E. Ottaviano, Multi criteria optimum design of manipulators, Bulletin of the Polish Academy of Sciences, Technical Sciences. 53(1) (2005) 9-18. 